\title{
SCUOLA, EMigRAZIONE ED \\ AlfabetizZAZIONE: IL CASO DI \\ Comuneglia in Alta Val di Vara
}

Davide Montino

ABSTRACT L'articolo ricostruisce, per sommi capi, la vicenda della scuola rurale di Comuneglia, nell'Appennino ligure. In particolare, mostra come l'emigrazione non abbia inciso solo sulla crescita di un diffuso bisogno di scrittura, ma anche sui processi di scolarizzazione. Tra Ottocento e Novecento, infatti, la scuola venne individuata, nelle comunità a forte vocazione migratoria, come un elemento decisivo per fornire gli strumenti necessari per affrontare il viaggio e la ricerca di un lavoro. Da questo punto di vista, pratiche simili erano già diffuse fin dal XVIII secolo, specialmente nelle aree montane, soggette all'emigrazione stagionale transfrontaliera. Il caso della scuola di Comuneglia, quindi, sembra inserirsi in questo contesto, evidenziando, tra l'altro, la figura di un educatore innovativo come Don Vincenzo Giannone.

PAROLE CHIAVE scuola; educazione; emigrazione; scrittura; alfabetizzazione. 
RESUMO O artigo reconstitui, em grandes linhas, a história da escola rural de Comuneglia, no Apenino da região Ligúria. Em particular, mostra que a emigração não somente influiu sobre o crescimento de uma necessidade difundida de aprendizagem da escritura, mas também sobre os processos de escolarização. Entre os séculos XIXe $X X$, com efeito, a escola foi apontada, nas comunidades com forte vocação migratória, como um elemento decisivo para fornecer os instrumentos necessários para enfrentarse a viagem e a busca de trabalho. Desse ponto de vista, iniciativas semelhantes existiam desde final do século XVIII, especialmente nas regiões montanhosas, atingidas pela emigração sazonal fronteiriça. O caso de Comuneglia, portanto, parece inserir-se nesse contexto, destacando-se, entre outros fatores, a figura de um educador inovador como o Padre Vincenzo Giannone.

PALAVRAS-CHAVE escola; educação; emigração; escritura; alfabetização.

ABSTRACT The article reconstructs, for sums heads, the vicissitude of the rural school of Comuneglia, situated in Liguria Appennino. In particular, extension as the emigration has not only affected the increase of a diffused need of writing, but also on the school mass processes. Between the 1800's and the 1900's, in fact, the school came characterized, in the communities strongly migratory vocation, like a decisive element in order to supply the instruments necessary in order to face the travel and to the search of a job. From this point of view, practical similar they were already diffuse since XVIII the century, especially in the rural areas. The case of the school of Comuneglia, therefore, seems to become part in this context, evidencing, among other things, the figure of an innovative pedagogist like Don Vincenzo Giannone.

KEYWORDS school; education; emigration; writing; literacy 


\section{Scritture d'emigrazione}

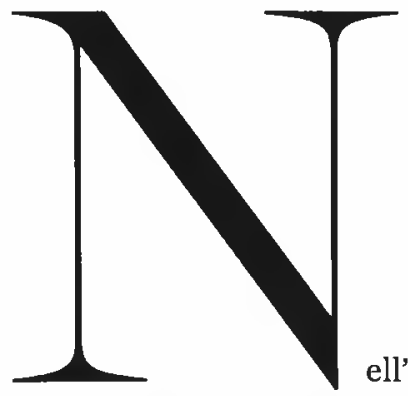

ell'ambito degli studi sui processi migratori sono emerse ingenti quantità di testimonianze scritte - lettere, diari, memorie - composte per lo più da persone comuni, appartenenti alle classi subalterne, ma non solo: contadini, operai, ma anche piccoli commercianti e artigiani, nonché imprenditori hanno così lasciato una mole considerevole di scritture, ${ }^{1}$ oggi a disposizione di studiosi e ricercatori che le possono interrogare in diversi archivi. ${ }^{2}$ Le scritture d'emigrazione testimoniano, molto spesso, di un linguaggio nuovo, che si crea dall'interazione tra la lingua madre, spesso il dialetto, la lingua del paese d'origine, e quella del paese d'arrivo. Anche quando i contenuti delle scritture mostrano un eloquente legame con la terra di provenienza, fino a rasentare un sentito patriottismo, spesso la lingua in cui sono vergate lascia trasparire ben maggiori scambi e sincretismi linguistici. Si legga, per esempio, quanto scrive Alisia, tra il $1936 \mathrm{e}$ il 1938, da Iquique al fratello Mario, per raccontargli alcune scene di vita cilena:

Carissimo Mario, Non ti puoi immaginare con quanto piacere leggo le lettere che scrivi a Lina specialmente saperti che ti ricordi con afetto e conservi ricordo di me, e di noi tutti, puoi stare sicuro che sei di onore e con afetto contraccambiato. hai raggione in lagnarti del mio silenzìcio ma certo tu sei tanto buono che saprai perdonarmi questo mio silencio che imparte è dovuto che trovarmi in colegio e non avendo da chi farmi correggere in cierta confidecia.

Ritardo in scriverti perché o quasi vergogna sollecitare l'aiuto delle Suore che parecchie sono Italiane.

Vorrei contarti tante cose mainverità la capacita mi manca non socome cominciare per nararti tutto quello che vorrei dirti. Siamo nella settimana primaverile cioè la festa

1. Sulle lettere di emigrazione si veda A. Gibelli e F. Caffarena, Le lettere degli emigranti, in P. Bevilacqua, A. De Clementi, E. Franzina (a cura di), Storia dell'emigrazione italiana - Partenze, Roma, Donzelli, 2001.

2. I maggiori centri italiani di raccolta, catalogazione estudio di "scritture popolari”, nei quali è possibile reperire anche scritture di emigrazione, sono L’Archivio Ligure della Scrittura Popolare dell'Università di Genova (www.dismec.unige.it/webalsp/alsp.htm), L'Archivio della Scrittura Popolare di Trento (www.museostorico.tn.it) e la Fondazione Archivio Diaristico Nazionale di Pieve S. Stefano (www.archiviodiari.it). 
del studente avevo voglia di andare a casa a Papà di questo mio desiderio malgrado sapevo que le suore sono un poco contrario dato che siamo vicino all'esame, papà che è tanto buono si interessa del mio desiderio e sabato verso le sei mi manda a prendere con una impiegata, casiera, ero veramente contenteta, pure era contento il papà avermi vicino. Rientrai domenica verso le 19, dopo aver visto il corso dei fiori ove presero parte i studianti, è stato discreto e mi sono divertita insieme a papà i Lina e tutti di casa.

Sono stata pure in casa el 4 novembre dato che in colonia si festeggia con vera entusiasmo. Il programma è stato bellissimo, messa en colegio dei padri saleziani in memoria dei caduti, alle 12. grande ricevimento in el circolo Italiano che è un locale veramente magnifico Papà andò perché esendo presidente del comitato coloniale aveva l'incarico di ricevere le autorità, e invitanti io Lina e Angelo siamo andati al l'ora precisa 12. vi sono stati magnifici discorsi.

Davano anche galletine champaña l'acto era magnifico imponente non vi erano meno di 500 persona, due grandi sale erano cuasi piccole per tanta gente, alle due si tornò a casa si pranzò e mi feci una scorpacciata di parsoti che erano squisito e da tanto tempo non li mangiavo.

Alle 19 tornai contenta in colegio.

Una novità quale primizia desidero cioè papà per prima volta lo visto con la camicia nera credi che se la mette stava così bene, sembrava più giovane quanto è buono non noi epure non lo dimostra à veramente un cuore d'oro non tralascia una domenica senza venirmi a vedere mi porta cioccolatini gallettine e frutta senza lasciarmi di mancare uova e marmelata $[. . .]^{3}$

È appena il caso di notare gli evidenti spagnolismi, non solo nell'ortografia, ma anche nel tentativo di rendere certi fonemi propri del castigliano. Ma quello che più colpisce è proprio la discrepanza tra un sentimento che si rivela spontaneo e sentito, quale la propria italianità, ed una lingua che è largamente debitrice del nuovo contesto in cui vive la bambina. Tanto più evidente, quanto più si pensi che Alisia era nata in Italia e aveva raggiunto solo in seguito la famiglia.

Nel quadro della storia della lingua, dunque, le scritture di emigrazione rappresentano un tassello importante, dal momento che restituiscono non solo dinamiche di alfabetizzazione, ma anche soluzioni linguistiche che si sono manifestate accanto alla formulazione delle lingue nazionali, cosi come è avvenuta nel corso del tempo. Sono un flusso di parole scritte che ha contribuito alla diffusione della lingua, o meglio che ha permesso una sorta di "traduzione" tra contesti espressivi differenti, dando vita molto spesso a forme linguistiche ibride, che lette oggi attestano tutta la forza e la profondità degli intrecci culturali che si sono determinati a seguito dei recessi migratori. Se è vero che anche attraverso l'insegnamento della lingua italiana sono passati i processi di 
nazionalizzazione messi in atto dallo Stato unitario tra Ottocento e Novecento, è altrettanto vero che in contesto migratorio quella stessa lingua si presta ad utilizzi più creativi, divenendo il modo espressivo della comunità migrante che la scrive.

Se l'emigrazione è stata indubbiamente un fattore di sviluppo della scrittura, allo stesso tempo sembra che abbia inciso, almeno per alcune aree italiane, anche sui processi di scolarizzazione, determinando - insieme al bisogno di scrittura - anche un bisogno di scuola sempre più impellente nel corso del XIX e del XX secolo.

\section{Emigrazione ed alfabetizzazione}

Come è stato notato, emigrare significava aver bisogno di comunicare con i familiari, tentare di ricomporre le fratture sociali ed esistenziali sottese all'abbandono della propria comunità, ma anche una necessità maggiore di saper leggere, per districarsi più agevolmente nel mercato del lavoro in paesi stranieri. In questo senso, nelle aree di emigrazione transfrontaliera, come ha mostrato Marina Roggero soprattutto per l'area alpina piemonetese, si sono sedimentate pratiche di alfabetizzazione fin dall'età moderna, le quali hanno inciso sui livelli più bassi di analfabetismo registrati in quelle zone fin dai primi censimenti unitari. ${ }^{4}$ Anche per la zona dell'Appenino ligure, Marco Porcella ha documentato, fin dai primi anni del XIX secolo, accanto a flussi migratori consistenti verso le Americhe, un bisogno sempre più crescente di alfabeto, l'unico strumento per mantenere rapporti con chi restava, ma anche per guidare spostamenti e strategie economiche e commerciali. ${ }^{5}$ Se passiamo da zone relativamente circoscritte alla realtà nazionale, non mancano gli stessi documenti coevi a testimoniare la stretta relazione tra emigrazione ed alfabetizzazione. Ad esempio, il relatore per gli Abruzzi e il Molise dell'inchiesta Faina (pubblicata tra il 1909 e il 1911) sulle condizioni dei contadini meridionali, Cesare Jarach, così riassumeva la sua indagine:

Dall'America vengonogli incitamenti alle mogli a mandare ifigliuoli a scuola; anch'essi potranno un giorno emigrare, e l'intimità dei segreti familiari sarà conservata e gli inganni dei ciurmatori saranno più facilmente evitati se essi sapranno agire da sé. ${ }^{6}$

Gli faceva eco Francesco Nitti, relatore per la Basilicata e le Calabrie, che si soffermava più dettagliatamente sulle cause dell'aumentato bisogno di scuole:

Tutte le deposizioni orali e scritte concordano in questo: che è l'emigrazione la causa principale dell'aumentata frequenza delle scuole. [...] Anzitutto il contadino

4. M. Roggero, L'alfabeto conquistato. Apprendere ed insegnare nell'Italia tra Sette ed Ottocento, Il Mulino, Bologna, 2000. 5. M. Porcella, Da girovaghi a emigranti. Lettere da Filadelfia, in P. Conti, G. Franchini, A Gibelli (a cura di), Storie digente comune nell'Archivio Ligure della Scrittura Popolare, EIG, Acqui Terme, 2002.

6.Inchiesta Faina, Relazione Jarach, p. 216, citato in E. De Fort, Scuola e analfabetismo nell Italia del 900, il Mulino, Bologna, 1995. 
emigrato in lontani paesi, assai più civili del suo, impara per propria esperienza ad apprezzare l'utilità dell'istruzione, del saper leggere e scrivere. Vede quanto meglio vengono pagati gli operai non analfabeti e quanto meno siano soggetti ad imbrogli, soprusi e truffe. Poi, per corrispondere con la sua famiglia, e per aver da essa notizie, sa quali inconvenienti procuri dover ricorrere ad estranei ai quali deve comunicare i fatti propri, e si affretta perciò ad ordinare alla sua donna che mandi i ragazzi a scuola a qualunque costo. ${ }^{7}$

In quest'ultima citazione è significativo anche il richiamo alla scuola. Se da un lato c'è un bisogno sociale diffuso di alfabetizzazione, dall'altro comincia a delinearsi l'esigenza anche di agenzie formali - ossia la scuola - in cui apprendere e fare pratica della scrittura. Da questo punto di vista non pare azzardata l'ipotesi che anche i processi migratori, in età contemporanea, abbiano influito sulle crescenti dinamiche di scolarizzazione, ed anzi ne siano stati in qualche modo i vettori soprattutto in aree periferiche, economicamente e culturalmente arretrate, come in larga misura era il Sud I talia tra Ottocento e Novecento. Allo stesso modo, nelle zone rurali del Nord, specialmente laddove l'agricoltura non era ancora stata interessata dalla modernizzazione delle pratiche e degli strumenti di coltivazione, e permanevano dunque bassi livelli di resa a fronte di uno sforzo produttivo notevole. Si pensi per esempio alle Langhe piemontesi, a vaste zone del Veneto, o all'aspro Appennino ligure. Non è raro, infatti, che proprio in tali zone si scoprano scuole fondate, fin dal Settecento, da membri della comunità emigrati, che rimettendo un po' delle loro risorse si sono interessati, accanto alla chiesa locale, di attrezzare proprio un luogo di apprendimento. Segno che il nesso tra istruzione ed emigrazione, almeno nella consapevolezza di chi aveva fatto quell'esperienza, era forte e poteva orientare ed agevolare le scelte di chi in futuro avesse voluto tentare la sorte emigrando.

Un caso paradigmatico di questo rapporto è, dunque, la scuola rurale di Comuneglia, situata proprio sull'Appennino ligure.

\section{La scuola di Comuneglia}

Un luogo dove antiche pratiche e nuovi modelli di mobilità geografica si incontrarono e diedero forma ad un diffuso bisogno di istruzione, come abbiamo detto, fu la montagna dell'Appennino ligure, dove, a partire almeno dagli anni Quaranta dell'Ottocento, si verificò un profondo processo di trasformazione della comunità contadina, che prese le forme dell'inurbamento e dell'industrializzazione, soprattutto intorno al polo urbano di Genova, e di una emigrazione 
transoceanica che toccò moltissimi dei componenti della comunità. Su queste montagne sorge il piccolo borgo di Comuneglia, nel comune di Varese Ligure, situato nell'Alta Val di Vara. I suoi abitanti, abituati già da secoli ad emigrare soprattutto in Francia, aggiunsero, dalla metà dell'Ottocento, alle loro solite mete i paesi dell'Europa centrale, come la Prussia, spingendosi poi verso Nord-est in Russia e verso Sud-est in Turchia, ma in particolar modo si diressero verso l'America, specie quella del Nord. Costretti a prendere la via dell'Oceano dalle difficili condizioni economiche - la montagna appenninica è aspra e spesso avara di frutti - ma anche in base a precise strategie di mobilità, ${ }^{8}$ gli abitanti di Comuneglia trovarono in Don Vincenzo Giannone ${ }^{9}$ (1827-1915) e nella sua scuola un valido aiuto, consistente nell'istruzione che lui aveva impartito a tanti piccoli scolari (e non solo) poi divenuti emigranti.

La figura di Don Giannone è una figura atipica. Uomo colto, amante della lettura e della scrittura, ispirato ad un moderno cristianesimo pedagogico di tipo spiritualista, che aveva i suoi referenti culturali in Tommaseo, Rosmini, Gioberti, egli vedeva l'istruzione come formazione morale dell'individuo, e pertanto aveva dedicato la vita all'insegnamento, in particolare nelle piccole comunità rurali. Anzi, dava molta più importanza al suo ruolo di maestro che a quello di sacerdote, tenendo in particolar modo a differenziare la sua scuola da quelle "scuole di campanile" troppo legate all'insegnamento scolastico tradizionale. A Don Giannone importava che i suoi alunni "imparassero comprendendo", in un rapporto costante con il mondo circostante e con il lavoro, specie quello agricolo. Sotto questo punto di vista, il parroco di Comuneglia appare un pedagogista moderno rispetto ai suoi tempi, attento alle esigenze dei fanciulli, volutamente distante da un modello educativo troppo legato alle pratiche religiose, ma non tanto per salvaguardare uno spazio pedagogico non confessionale - che lo avrebbe posto al di fuori del contesto in cui invece viveva ed operava - quanto per poter sperimentare forme nuove di istruzione, in grado di formare uomini sì rispettosi della chiesa e dei suoi insegnamenti, ma anche capaci di cavarsela da soli, potendo contare su quello che sapevano ed avevano imparato.

Se l'opera del maestro era compiuta con estrema attenzione e dovizia, da sola non basta però a spiegare l'accoglienza e il riscontro trovato presso la comunità. Non bisogna dimenticare, infatti, che la scuola era perlopiù vista come un orpello accessorio, e leggere e scrivere e far di conto non erano che le minime capacità strumentali che nemmeno tutti possedevano, e sentivano il bisogno di possedere. Quello che contraddistinse l'esperienza di Don Giannone è che questo suo esemplare impegno si innestò su una diffusa domanda di istruzione, che andava aumentando mano a mano che si intensificava il processo migratorio, e che gli emigranti si rendevano conto dell'importanza che aveva il saper padroneggiare l'alfabeto, per scrivere ma anche per leggere le lettere che ricevevano o i giornali, e per sentirsi ancora parte della comunità d'origine. Senso di appartenenza abbondantemente testimoniata dalle tante sottoscrizioni che facevano per donare alla chiesa un altare, una statua o una riparazione essenziale. Ed è proprio questo intreccio ad apparire significativo e a far emergere il bisogno di scrittura come un potente fattore di sviluppo

8. Cfr. A. Gibelli, La risorsa America, in P. Rugafiore, A. Gibelli (a cura di), Storia d'Italia, Le regioni, dall'unità a oggi. La Liguria, Torino, Einaudi, 1994.

9. Su Don Vincenzo Giannone cfr. M. Podestà, L'esperienza dell'emigrazione attraverso l'Epistolario di Don Giannone, Tesi di Laurea, Facoltà di Lettere e Filosofia, A.A.1995/96. 
della scolarizzazione, almeno nella prospettiva locale che qui si presenta. Senza il determinarsi di cospicui flussi migratori, infatti, sembra ben più difficile immaginare una così forte richiesta di alfabeto e di scuola. Tant'è che nelle stesse intenzioni delle autorità locali, le quali avevano fin dall'inizio sostenuto il progetto di una scuola, l'insegnamento del maestro era particolarmente da apprezzare per l'amore che infondeva per "l'arte del contadino", in modo da porre rimedio "a due grandi piaghe che affliggono la nostra cara patria: dell'emigrazione e della smania di affluire ai grandi centri per trovar lavoro" 10 Insomma, le intenzioni che avevano sorretto la fondazione della scuola andavano proprio nella direzione opposta alla funzione che aveva assunto col tempo. Pensata per legare, mediante una precisa istruzione agricola, gli abitanti di Comuneglia al loro paese, in realtà la scuola era spesso il primo passo in direzione delle Americhe: era il luogo in cui si affinavano gli strumenti comunicativi che si sarebbero rivelati utili e fondamentali una volta partiti per l'Oceano.

La scuola di Don Giannone visse dal 1852 al 1905, interessando un numero di scolari che variava, da anno ad anno, tra i 70 e i 90 alunni, quasi la totalità dei bambini in età scolare. Fino al 1883 fu attiva anche la scuola serale e festiva, per gli adulti, le donne e quei bambini che per diversi motivi - spesso l'impiego in lavori domestici o agricoli - non potevano frequentare con assiduità.

Dato il cospicuo numero di alunni, ed anche il poco spazio a disposizione per le aule, il metodo adottato in questa scuole era quello del "mutuo appoggio" conosciuto anche come metodo "lancasteriano", che prevedeva l'utilizzo dei migliori alunni in supporto al maestro, i quali ricevevano anche una piccola paga. Inoltre la scuola non era fissa in un luogo, ma toccava un po' tutte le frazioni della borgata, in modo da raggiungere gli allievi ovunque fossero. Una scuola, insomma, che era articolata per quante erano le frazioni, raggiungendo i suoi allievi laddove si trovavano. Anche questo un elemento non del tutto inconsueto, poiché spesso i maestri nei primi decenni unitari erano costretti ad inseguire gli scolari nelle loro case, facendo lezione nelle stalle, in cui spesso finivano per essere coinvolti anche gli adulti. Quello che appare più interessante, se mai, è che la mobilità della scuola di Don Giannone non rispondeva solo ad una necessità - raggiungere quegli studenti che non avrebbero in altro modo partecipato alla scuola-, ma era frutto di un progetto ben definito, consistente nel formulare una proposta di istruzione che oggi definiremmo "flessibile" proprio in virtù dell'alta richiesta di istruzione diffusa tra gli abitanti di Comuneglia.

Che l'esperienza di questa scuola fosse qualcosa di particolare lo testimonia il rapporto tra Don Giannone e i suoi allievi, il quale durava oltre la scuola, come documentano le lettere che molti di essi gli inviarono, conservate tra le tante missive (più di ottocento) dell'epistolario del parroco che si trovano in copia all'Archivio Ligure della Scrittura Popolare. Don Giannone teneva particolarmente a questo rapporto, sentendosi quasi un padre per i suoi ex allievi e facendo da collettore tra gli emigrati e la loro "patria", che essi identificavano con il paese di origine. Da queste lettere si vede chiaramente la capacità degli emigrati di mantenere saldi i rapporti con la comunità d'origine e tra di essi (sono sparsi tra l'Europa, la California, l'Argentina e il Perù), quasi che le 
distanze fossero annullate dalle lettere attraverso cui comunicavano. Si comprende, allora, e fino in fondo, quel bisogno di istruzione che abbiamo visto manifestarsi nella comunità di Comuneglia, e tutta la valenza sociale ma anche psicologica del saper scrivere. Una volta attraversato l'Oceano, infatti, solo la parola scritta poteva far da tramite tra la propria origine e il nuovo mondo (fisico ma anche e soprattutto mentale) e creare addirittura un senso di apparenza molto forte tra i comunegliesi sparsi nel mondo. ${ }^{11}$ Esemplare a questo proposito è la richiesta di Don Giannone agli emigrati a Stockton di spedirgli per il suo cinquantenario di sacerdozio una foto di gruppo, "come han fatto quelli di Buonos Aiers, e son dietro a fare quelli di Lima, di Giacquil e spero di New York e di Dai ton" 12

Da quanto abbiamo sommariamente visto la scuola di Comuneglia si presta a mettere in evidenza alcuni aspetti: essa è il riflesso di un bisogno di scrittura che scaturisce dai processi migratori, $\mathrm{ma}$ anche di pratiche di istruzione popolare, ed infine di un senso di appartenenza che supera la dimensione del contatto fisico. Proprio grazie alla sua funzione di mediatore, Don Giannone tiene insieme gli ex alunni, che continuano a percepirsi come elementi di una comunità che potremmo definire virtuale, nonostante siano sparsi in tutto il mondo: la scuola diventa il luogo di una memoria che resiste al tempo e allo spazio, e la lingua scritta lo strumento di una comunicazione che tiene insieme le esperienze degli emigranti. Non solo e non più un mezzo per resistere alla destabilizzazione propria dell'emigrazione, o per gestire gli affari e i rapporti nel paese d'origine, ma elemento strutturale di un'appartenenza che resiste agli urti della modernità. Tutto ciò non sarebbe stato possibile senza la scuola di Don Giannone.

\section{Bibliografia}

A. Bartoli Langeli, La scrittura dell'italiano, Il Mulino, Bologna, 2000

E. De Fort, Scuola e analfabetismo nell'Italia del '900, Il Mulino, Bologna, 1995

A. Gibelli e F. Caffarena, Le lettere degli emigranti, in P. Bevilacqua, A. De Clementi, E. Franzina (a cura di), Storia dell'emigrazione italiana - Partenze, Roma, Donzelli, 2001

A. Gibelli, La risorsa America, in P. Rugafiore, A. Gibelli (a cura di), Storia d'Italia, Le regioni, dall'unità a oggi. La Liguria, Torino, Einaudi, 1994

D. Lercari, La "Patria" e la "Merica". Epistolari di emigranti liguri, in P. Conti, G. Franchini, A. Gibelli (a cura di), Storie di gente comune nell'Archivio Ligure della Scrittura Popolare, EIG, Acqui Terme, 2002

M. Porcella, Da girovaghi a emigranti. Lettere da Filadelfia, in P. Conti, G. Franchini, A. Gibelli (a cura di), Storie di gente comune nell'Archivio Ligure della Scrittura Popolare, EIG, Acqui Terme, 2002

M. Roggero, Apprendere ed insegnare nell'Italia tra Sette ed Ottocento, Il Mulino, Bologna, 2000

11. Sui rapporti tra la terra natia e l'America si veda D. Lercari, La "Patria" ela "Merica". Epistolari diemigrantiliguri, in P. Conti, G. Franchini, A. Gibelli (a cura di), Storie di gente comune, op cit. In particolare sui rapporti di Don Giannone con i suoi ex allievi, in quest'ottica, pp. 63-64.

12. Epistolario Don Giannone, lettera a Guglielmo Ghiggeri, 10 febbraio 1901. 